\title{
Cleavage Stage versus Blastocyst Stage Embryo Transfer: A Controlled Randomized Study
}

Eman A. Hassan, Mohamed Shehata

International Islamic Center for Population Studies and Research, Assisted Reproduction Unit, Al-Azhar University. International Islamic Centre for Population Studies and Researches (IICPR) Al-Azhar University
Dr.Eman Anwar Hassan / International Islamic Centre for Population Studies and Researches (IICPR) Al-Azhar University, Cairo, Egypt.Tel: (+2) 01221354112 . E-mail: emanembryology@gmail.com

\section{$\underline{\text { Abstract }}$}

Background: Both cleavage-stage and blastocyst-stage embryo transfer policies have advantages and drawbacks. The number of embryos transferred, however, is a crucial parameter that needs to be considered before attempting any comparison. Advances in the dynamics of embryo culture allow us to culture embryos to the blastocyst stage on day 5). Prolonging the duration of culture to Day 5 allows chromosomally competent embryos to develop to the blastocyst stage and permits selection of embryos that has the potential for continued development under embryonic genomic control (1). In addition, selection of Day 5 embryos has the advantage of physiological synchronization with the uterine endometrial, thereby resulting in better pregnancy rates (2).

Objective: The aim of this study is comparing intracytoplasmic sperm injection (ICSI) results: fertilization rate, Cleavage rate ( number\& quality of embryos) and clinical pregnancy rate, to evaluate the efficacy of blastocyst transfer in comparison with cleavage stage transfer ( 4 cell stage \& 8 cell stages).

Patients and Methods: 600 patients aged 25-40 years, who were scheduled for intracytoplasmic sperm injection cycle from Jan 2014 to Dec 2015, were recruited in the study. After assessment of fertilization, patients were divided randomly into three study groups, day 2, day 3 and day 5 embryo transfer groups having 200 patients each. Primary outcomes, clinical pregnancy rate and implantation rate were evaluated.

Results: all the groups were similar for age, indication and number of embryos transferred. Clinical pregnancies after blastocyst transfer were significantly higher $51 \%$ compared to cleavage stage embryo transfer $37 \%$ (day 2), $48.5 \%$ (day 3 ).

Conclusion: Blastocyst transfer having high implantation rate and clinical pregnancy rate leads to reduction in multiple pregnancies.

Key Words: Cleavage stage - Blastocyst - Embryo Transfer - ICSI.

\section{Introduction}

The fledgling era of in vitro fertilization (IVF) from 1980 to the mid1990 s, was characterized by relatively static success rates of around $20 \%$ pregnancy rates (3). The past decade however, has given rise to exciting advances in ovarian stimulation, cell culture and embryo transfer techniques that have culminated in significant overall improvements in successful pregnancies (4). This is evident in the annual statistical reports from different areas around the world (5). Previous study demonstrated a doubling of pregnancy rate per embryo transfer cycle from 1994 to 2003 despite a decrease in the mean number of embryos transferred (6). The contribution of embryo culture to 
these improvements is the focus of this Cochrane review of cleavage stage versus blastocyst stage embryo transfer. With the introduction of a variety of commercial preparations of sequential media in the late 1990s, the IVF industry witnessed an explosion of worldwide interest in blastocyst culture, with most clinics conducting research into its application in their own setting. As a result a substantial volume of publications followed (7). These included conflicting trials and debates about the merits and drawbacks of extended culture. (8). A lack of strong consensus about the best practice for blastocyst culture has not aided by the fact that many of the trials were not prospectively randomized and/or were under powered (9). The need for an evidence-based approach using metaanalysis of small trials was, therefore, required to assist in deciphering the overall effect of blastocyst culture to help identify patient subsets and practices that might best benefit from this approach. (10). Blastocyst culture is not novel; indeed, the very first report of an IVF pregnancy was from a transferred blastocyst (11).

Despite this, cleavage stage transfer was adopted as standard global practice early in the history of IVF because of: a) the low developmental rate of embryos cultured past this stage and b) unlike other primates, human embryos have the unusual propensity to survive when replaced prematurely into the uterus (12). However, as knowledge of embryo metabolic requirements expanded, so did the range of more advanced culture media (13) and co-culture techniques (14). The most dramatic was the understanding that the in vitro environment in which an early cleavage stage embryo grows best in is different from that of a blastocyst stage (15). This led to the evolution of stage-specific or sequential media (G1/G2), by Gardner in 1998 (16); embryos are transferred on Day 3 from a medium containing low concentrations of glucose and one or more amino acids to a medium containing higher concentrations of glucose and a wider range of amino acids (17). At this stage, the embryo undergoes cell compaction and genomic activation so that the embryo is no longer under the control of transcripts RNA messages of maternal origin (18). With the application of stage-specific media, there have been reports of blastocyst development and implantation rates as high as $60 \%$ to $65 \%$ (19).

There are two central arguments why blastocyst culture has purported advantages over traditional cleavage stage embryo transfer. Firstly, it has long been recognized that it is physiologically premature to expose early-stage embryos to the uterine environment, particularly one that has been subjected to superovulation and thus high levels of estrogen (20). In vivo, embryos travel through the fallopian tubes and do not reach the uterus before the morula stage (16 cell or compacted), which equates to at least Day 4 of in vitro culture (21). The uterus provides a different nutritional environment from the oviduct; therefore, it is postulated that this may cause stress on the embryo and result in reduced implantation potation rate (22). There is also evidence of a significant reduction in uterine pulsation at the time when blastocysts are transferred and therefore less chance that embryos can be expelled (23). The second argument for blastocyst culture is in their innately higher implantation potential compared with early cleavage embryos. As a consequence of self-selection, it is postulated that only the most viable embryos are expected to develop into blastocysts (24). Arguments against blastocyst culture are largely related to this process of selfselection. Couples undergoing blastocyst culture are expected to have a higher incidence of: a) being cancelled due to failed embryo development to blastocyst (25) and b) having fewer embryos for cryopreservation (freezing) (26).

There is also the question of how scientists can be so certain that any given; Day 3 embryo has the ability to become a viable blastocyst in vivo, but not in vitro. Based on the very wide range of blastulaion rates reported, there is evidence that not all clinical and laboratory environments are equal, despite identical sequential media being used. This is an obvious compounding factor when performing a meta-analysis. Variables such as number of incubators, gas mix, culture ware quality control, and the superovulation regimen have all been reported to have an impact of blastocyst culture outcomes $(27,28)$.

For this reason there may be an argument for introducing a minimum Day 2 to 3 implantation rate (that is approximately $20 \%$ ) for trial inclusion criteria, but this may differ depending on the overall patient prognosis for each trial (29). Single embryo transfers for selected patient groups are now considered standard practice in many clinics throughout the world (30). The importance of selecting the single most viable embryo for transfer has intensified the search for improving the assessment of the quality of embryos. 
Performing blastocyst culture may offer one of those mechanisms (31). Advocates of blastocyst culture are confident that only the most viable embryos will survive the extended culture to Day 5 to 6 . This would result in a higher probability of implantation and require fewer embryos to be transferred, thereby lowering the costly multiplebirth rate (32).

Critics of the approach express concern at the increased incidence of women failing to have embryos available for transfer (33), although the day of patient recruitment into the blastocyst program is crucial to this argument. It is important to be aware that clinic policies may differ on the minimum criteria for blastocyst culture and the day on which this decision is made (for example number of follicles, fertilized eggs, eight-cell embryos on Day 3) .It is also yet to be clarified if there are patient groups for whom blastocyst culture is disadvantageous. And most importantly, does blastocyst culture achieve the primary aim of providing the sub fertile couple with a normal, healthy baby (34).

\section{Patients and Methods}

This prospective clinical trial study was carried out in the Assisted Reproductive Unit at the International Islamic Centre for Population Studies and Research (IICPSR), Al-Azhar University, Cairo, Egypt. All couples were subjected to detailed explanation about the procedure. All cases were clinically evaluated. A full general and local examination of both couples was done. Routine investigations and hormonal assays were requested according to our unit protocol. Study includes six hundred couples; female aged 25-40 years undergoing intracytoplasmic sperm injection (ICSI) cycle from January 2014 to December 2015. Inclusion criteria set namely, 2-10 years of infertility, having minimum five Oocytes at Oocytes pick up, endometrial thickness of 8 $\mathrm{mm}$ and more indicating good ovarian response, having normal uterine cavity and basal folliclestimulating hormone (FSH) $<10 \mathrm{~m} \mathrm{IU} / \mathrm{ml}$.

\section{Patient stimulation and oocyte retrieval}

Patients were put on long protocol, gonadotropinreleasing hormone $(\mathrm{GnRH})$-agonist started on Day 21.(from previous cycle), and daily doses given subcutaneously till Day 3 of next cycle. Hormonal evaluation: Serum FSH, luteinizing hormone (LH), Estradiol (E2) and transvaginal sonography was done on Day 3 to confirm down regulation.

Induction with recombinant FSH (rFSH) was started once pituitary down regulation was confirmed. The dose schedule was modified according to parameters like body mass index (BMI), previous response and ovarian reserve estimates and was given for four days (Day 3-6). Follicular monitoring was initiated on Day 7 of cycle and further doses of rFSH were given according to follicle size and continued till Day 11. Women were scheduled for Oocytes retrieval when at least five follicles reached $18 \mathrm{~mm}$ size and injection human chorionic gonadotropin (HCG) 10,000 IU was given. Transvaginal sonography guided Oocytes retrieval was then planned 36 hours after $\mathrm{HCG}$, which was performed under short general anesthesia.

\section{Sperm preparation:}

All semen samples liquefy for 15 to 30 minutes in an incubator. Sperm was prepared using sperm wash media. One-half to $2 \mathrm{~mL}$ of raw semen was layered and the preparations were centrifuged at $300 \mathrm{~g}$ for 20 minutes. After centrifugation, the pellets from each tube were collected into $5 \mathrm{~mL}$ of culture medium and centrifuged at $1800 \mathrm{~g}$ for ICSI for 10 minutes. Fertilization was confirmed by the presence of two pronuclei and two polar bodies on day 1 .

\section{Embryo culture and development}

The retrieved Oocytes were then incubated for 1 hour in global fertilization media and then, depending on maturity of Oocytes, ICSI was performed. Denudation of Oocytes was carried out chemically \&mechanically before ICSI was performed. Injected Oocytes were incubated overnight in global total media in a triple gas incubator (Labotect) and observed after 16-18 hours post insemination for fertilization. The fertilized Oocytes were then transferred into a cleavage medium and incubated. Embryos were observed on Day 1(2cell stage) and transfer was scheduled according to quality of embryos:

Group 1: Included patients undergoing embryo transfer on Day 2(200 case).

Group 2: Included patients undergoing embryo transfer on Day 3(200 case).

Group 3: Included patients undergoing embryo transfer on Day 5(200 case). 
All transfers were performed using Labotect catheter. Luteal support was given in form of micronized vaginal progesterone in a dose of 200 $\mathrm{mg}$ thrice-daily for 18 days post retrieval. Serum B-HCG was performed on Day 15 following embryo transfer and if positive then transvaginal sonography was performed 15 days later to detect and confirm intrauterine pregnancy. The study population in three groups was comparable.

\section{Statistical Analysis:}

Data are presented as means \pm standard deviation. For statistical analysis the $\chi 2$ and Fisher's exact tests were used. Statistical significance was set at $\mathrm{p}<0.05$.

\section{$\underline{\text { Results }}$}

This randomized, prospective study was conducted in assisted reproduction unit at the International Islamic Centre for Population Studies and Research (IICPSR), Al-Azhar University, Cairo, Egypt. On 600 patients aged 25-40 years undergoing intracytoplasmic sperm injection (ICSI) cycle from Jan 2014 to Dec 2015. When five or more Grade A embryos were observed on Day 2 lof culture, patients were divided randomly into three study groups, day 2 embryo transfer, day 3 embryo transfer and day 5 embryo transfer group having 200 patients each.

Table 1: it shows the age distribution among all studied groups $(n=600)$.

\begin{tabular}{|c|c|c|c|c|c|c|}
\hline \multirow{4}{*}{$\begin{array}{c}\text { Age } \\
\text { groups }\end{array}$} & Day 2 & Day 3 & Day 5 & \multicolumn{2}{c|}{ ANOVA } \\
\cline { 2 - 7 } & Mean \pm SD & Mean \pm SD & Mean \pm SD & F & P-value \\
\hline \multirow{4}{*}{ Age } & $\mathbf{2 1 - 2 5}$ & $23.960 \pm 1.160$ & $23.380 \pm 1.354$ & $22.700 \pm 1.644$ & 10.143 & $<0.001^{*}$ \\
\cline { 2 - 7 } & $\mathbf{2 6 - 3 0}$ & $28.140 \pm 1.370$ & $27.720 \pm 1.415$ & $27.620 \pm 1.227$ & 2.121 & 0.124 \\
\cline { 2 - 7 } & $\mathbf{3 1 - 3 5}$ & $33.360 \pm 1.290$ & $33.360 \pm 1.987$ & $33.100 \pm 1.460$ & 0.436 & 0.647 \\
\cline { 2 - 7 } & $\mathbf{3 6 - 4 0}$ & $38.440 \pm 1.459$ & $37.940 \pm 1.420$ & $38.480 \pm 1.129$ & 2.505 & 0.085 \\
\hline
\end{tabular}

There was no statistically significant difference among the studied groups as regards the age distribution except in the age group $21-25$ years $\left(\mathrm{P}<0.001^{*}\right)$.

Table 2:it shows distribution of collected oocytes among all studied groups ( $n=600)$

\begin{tabular}{|c|c|c|c|c|c|c|}
\hline & Age & Day 2 & Day 3 & Day 5 & \multicolumn{2}{|c|}{ ANOVA } \\
\cline { 2 - 7 } & groups & Mean \pm SD & Mean \pm SD & Mean \pm SD & F & P-value \\
\hline \multirow{4}{*}{$\begin{array}{c}\text { collected } \\
\text { oocytes }\end{array}$} & $\mathbf{2 1 - 2 5}$ & $6.880 \pm 3.008$ & $8.000 \pm 3.010$ & $10.320 \pm 2.325$ & 19.638 & $<0.001^{*}$ \\
\cline { 2 - 7 } & $\mathbf{2 6 - 3 0}$ & $7.240 \pm 3.223$ & $6.600 \pm 1.927$ & $9.900 \pm 2.565$ & 22.207 & $<0.001^{*}$ \\
\cline { 2 - 7 } & $\mathbf{3 1 - 3 5}$ & $7.480 \pm 3.887$ & $7.100 \pm 3.346$ & $10.440 \pm 3.418$ & 13.203 & $<0.001^{*}$ \\
\cline { 2 - 7 } & $\mathbf{3 6 - 4 0}$ & $4.280 \pm 2.564$ & $6.080 \pm 3.155$ & $10.520 \pm 1.657$ & 80.294 & $<0.001^{*}$ \\
\hline
\end{tabular}

There was a highly significant difference among all the studied groups as regards the number of collected oocytes $(\mathrm{P}<0.001)$.

Table 3: it shows the number of injected oocytes among all studied groups $(n=600)$.

\begin{tabular}{|c|c|c|c|c|c|c|}
\hline & Age & Day 2 & Day 3 & Day 5 & \multicolumn{2}{|c|}{ ANOVA } \\
\cline { 2 - 7 } & groups & Mean \pm SD & Mean \pm SD & Mean \pm SD & F & P-value \\
\hline \multirow{4}{*}{$\begin{array}{c}\text { injected } \\
\text { oocytes }\end{array}$} & $\mathbf{2 1 - 2 5}$ & $4.480 \pm 2.063$ & $4.640 \pm 1.816$ & $7.600 \pm 1.917$ & 41.253 & $<0.001^{*}$ \\
\cline { 2 - 7 } & $\mathbf{2 6 - 3 0}$ & $5.000 \pm 2.167$ & $4.640 \pm 1.575$ & $7.520 \pm 2.613$ & 26.383 & $<0.001 *$ \\
\cline { 2 - 7 } & $\mathbf{3 1 - 3 5}$ & $4.780 \pm 2.288$ & $4.280 \pm 2.204$ & $7.340 \pm 1.586$ & 32.053 & $<0.001 *$ \\
\cline { 2 - 7 } & $\mathbf{3 6 - 4 0}$ & $3.140 \pm 1.414$ & $4.500 \pm 2.652$ & $8.800 \pm 1.309$ & 121.856 & $<0.001 *$ \\
\hline
\end{tabular}

There was a highly significant difference among all the studied groups as regards the number of injected oocytes $(\mathrm{P}<0.001)$. 
Table 4: it shows the number of fertilized oocytes among all studied groups $(n=600)$.

\begin{tabular}{|c|c|c|c|c|c|c|}
\hline & Age & Day 2 & Day 3 & Day 5 & \multicolumn{2}{|c|}{ ANOVA } \\
\cline { 2 - 7 } & groups & Mean \pm SD & Mean \pm SD & Mean \pm SD & F & P-value \\
\hline \multirow{4}{*}{ Fertilization } & $\mathbf{2 1 - 2 5}$ & $3.700 \pm 1.8323$ & $3.380 \pm 1.510$ & $6.542 \pm 1.750$ & 50.860 & $<0.001 *$ \\
\cline { 2 - 7 } & $\mathbf{2 6 - 3 0}$ & $3.460 \pm 1.460$ & $3.660 \pm 1.479$ & $6.520 \pm 1.555$ & 65.247 & $<0.001 *$ \\
\cline { 2 - 7 } & $\mathbf{3 1 - 3 5}$ & $3.660 \pm 1.573$ & $3.560 \pm 1.875$ & $6.560 \pm 1.541$ & 52.065 & $<0.001 *$ \\
\cline { 2 - 7 } & $\mathbf{3 6 - 4 0}$ & $2.780 \pm 1.266$ & $3.360 \pm 1.626$ & $7.760 \pm 0.431$ & 250.937 & $<0.001 *$ \\
\hline
\end{tabular}

There was a highly significant difference among all the studied groups as regards the number of fertilized oocytes $(\mathrm{P}<0.001)$.

Table 5: it shows the number of Grade A embryos among the studied groups

\begin{tabular}{|c|c|c|c|c|c|c|c|c|c|}
\hline \multirow[t]{2}{*}{ Embryos } & \multirow{2}{*}{$\begin{array}{c}\text { Age } \\
\text { groups }\end{array}$} & \multicolumn{2}{|c|}{ Day 2} & \multicolumn{2}{|c|}{ Day 3} & \multicolumn{2}{|c|}{ Day 5} & \multicolumn{2}{|c|}{ Chi-square } \\
\hline & & $\mathbf{N}$ & $\%$ & $\mathbf{N}$ & $\%$ & $\mathbf{N}$ & $\%$ & $\mathrm{X} 2$ & P-value \\
\hline \multirow{4}{*}{ Grade A } & $21-25$ & $103 / 149$ & 69.1 & $107 / 155$ & 69 & $168 / 280$ & 60 & \multirow[t]{4}{*}{14.6} & \multirow[t]{4}{*}{$\mathrm{P}<0.001$ * } \\
\hline & $26-30$ & $105 / 152$ & 69.0 & $107 / 150$ & 71.3 & $228 / 300$ & 76 & & \\
\hline & 31-35 & $90 / 150$ & 60 & $99 / 145$ & 68.2 & $206 / 287$ & 71.7 & & \\
\hline & $36-40$ & $59 / 100$ & 59 & $84 / 140$ & 60 & $135 / 230$ & 58.6 & & \\
\hline
\end{tabular}

There was a highly significant difference among the studied groups as regards number of Grade A embryos $\left(\mathrm{P}<0.001^{*}\right)$.

Table 6: it shows the number of Grade B embryos among the studied groups

\begin{tabular}{|c|c|c|c|c|c|c|c|c|c|}
\hline \multirow{2}{*}{ Embryos } & Age & \multicolumn{2}{|c|}{ Day 2 } & \multicolumn{2}{c|}{ Day 3 } & \multicolumn{2}{c|}{ Day 5 } & \multicolumn{2}{c|}{ Chi-square } \\
\cline { 2 - 9 } & groups & $\mathbf{N}$ & $\mathbf{0}$ & $\mathbf{N}$ & $\mathbf{\%}$ & $\mathbf{N}$ & $\mathbf{\%}$ & X2 & P-value \\
\hline \multirow{4}{*}{ Grade B } & $\mathbf{2 1 - 2 5}$ & $35 / 149$ & 23.4 & $32 / 155$ & 20.6 & $65 / 280$ & 23.2 & 11.2 & $0.01 *$ \\
\cline { 2 - 10 } & $\mathbf{2 6 - 3 0}$ & $36 / 152$ & 23.6 & $36 / 150$ & 24.0 & $70 / 300$ & 23.3 & & \\
\cline { 2 - 9 } & $\mathbf{3 1 - 3 5}$ & $39 / 150$ & 26.0 & $40 / 145$ & 27.5 & $74 / 287$ & 25.7 & & \\
\cline { 2 - 9 } & $\mathbf{3 6 - 4 0}$ & $29 / 100$ & 29.0 & $47 / 140$ & 33.5 & $66 / 230$ & 28.6 & & \\
\hline
\end{tabular}

There was a significant difference among the studied groups as regards the number of Grade B embryos $\left(\mathrm{P}=0.01^{*}\right)$.

Table 7: it shows the number of Grade $\mathrm{C}$ embryos among the studied groups

\begin{tabular}{|c|c|c|c|c|c|c|c|c|c|}
\hline \multirow{2}{*}{ Embryos } & \multirow{2}{*}{$\begin{array}{c}\text { Age } \\
\text { groups }\end{array}$} & \multicolumn{2}{|c|}{ Day 2} & \multicolumn{2}{|c|}{ Day 3} & \multicolumn{2}{|c|}{ Day 5} & \multicolumn{2}{|c|}{ Chi-square } \\
\hline & & $\mathbf{N}$ & $\%$ & $\mathbf{N}$ & $\%$ & $\mathbf{N}$ & $\%$ & $\mathrm{X} 2$ & P-value \\
\hline \multirow{4}{*}{ Grade C } & $21-25$ & $11 / 149$ & 7.4 & $16 / 155$ & 10.4 & $47 / 280$ & 16.8 & \multirow[t]{4}{*}{2.1} & \multirow[t]{4}{*}{0.1} \\
\hline & $26-30$ & $11 / 152$ & 7.3 & $7 / 150$ & 4.7 & $2 / 300$ & 0.7 & & \\
\hline & $31-35$ & $21 / 150$ & 14 & $6 / 145$ & 4.2 & $7 / 287$ & 2.5 & & \\
\hline & $36-40$ & $12 / 100$ & 12 & $9 / 140$ & 6.5 & $29 / 230$ & 12.7 & & \\
\hline
\end{tabular}

There was no significant difference among the studied groups as regards the number of Grade $\mathrm{C}$ embryos $(\mathrm{P}=0.1)$. 
Table 8: it shows the number of pregnant women among the studied groups

\begin{tabular}{|c|c|c|c|c|c|c|c|c|c|}
\hline \multirow[t]{2}{*}{ Embryos } & \multirow{2}{*}{$\begin{array}{c}\text { Age } \\
\text { groups }\end{array}$} & \multicolumn{2}{|c|}{$\begin{array}{c}\text { Day 2 } \\
(74=37 \%)\end{array}$} & \multicolumn{2}{|c|}{$\begin{array}{c}\text { Day } 3 \\
(97=48.5 \%)\end{array}$} & \multicolumn{2}{|c|}{$\begin{array}{c}\text { Day } 5 \\
(102=51 \%)\end{array}$} & \multicolumn{2}{|c|}{ Chi-square } \\
\hline & & $N=50$ & $\%$ & $\mathrm{~N}=\mathbf{5 0}$ & $\%$ & $\mathrm{~N}=\mathbf{5 0}$ & $\%$ & $\mathbf{X} 2$ & P-value \\
\hline \multirow{4}{*}{$\begin{array}{c}+ \text { ve } \\
\text { pregnancy }\end{array}$} & $21-25$ & 17 & 34 & 25 & 50 & 26 & 52 & \multirow[t]{4}{*}{9} & \multirow[t]{4}{*}{$\mathrm{P}=0.002 *$} \\
\hline & $26-30$ & 21 & 42 & 29 & 58 & 30 & 60 & & \\
\hline & $31-35$ & 20 & 40 & 24 & 48 & 28 & 56 & & \\
\hline & $36-40$ & 16 & 32 & 19 & 38 & 18 & 36 & & \\
\hline
\end{tabular}

There was a significant difference among the studied groups as regards the number of pregnant women $(\mathrm{P}=0.002 *)$.

\section{$\underline{\text { Discussion }}$}

Blastocyst transfer is gaining popularity nowadays due to its higher implantation rates and clinical pregnancy rates. This study, which was performed in the Assisted Reproduction Unit at the International Islamic Centre for Population Studies and Research (IICPSR), Al-Azhar University, Cairo, Egypt, evaluated the intracytoplasmic sperm injection (ICSI) results: fertilization rate, Cleavage rate (number\& quality of embryos) and clinical pregnancy rate, to assess the efficacy of blastocyst transfer in comparison with cleavage stage transfer ( 4 cells \& 8 cell stages).

In this study, there was no significant difference between both the studied groups in terms of age distribution, duration of infertility, indication of infertility and type of infertility except in the age group 21-25 years, $\mathrm{P}<0.001$ (Table 1), This was in agreement with the study conducted by Van der Auwera et al. (35).

There was a highly significant difference among the studied groups regarding the number of collected oocytes, $\mathrm{P}<0.001$ (Table 2). Also, there was a highly significant difference among the studied groups as regards the number of injected oocytes $(\mathrm{P}<0.001)$ (Table 3$)$. There was a highly significant difference among the studied groups regarding the number of fertilized oocytes , $\mathrm{P}<$ 0.001 (Table 4). There was a highly significant difference among the studied groups as regards the number of Grade A embryos $(\mathrm{P}<0.001)$ (Table 5).

There was a significant difference among the studied groups as regards the number of Grade $\mathrm{B}$ embryos, $\mathrm{P}=0.01$ * (Table 6), There was no significant difference among the studied groups as regards the number of Grade $\mathrm{C}$ embryos, $\mathrm{P}=$ 0.1 (Table 7). There was a significant difference among the studied groups as regards the number of pregnant women, $\mathrm{P}=0.002 *$ (Table 8).

The results of our study were similar to the study of Mangalraj et al. (36). Two studies both published in 2004 evaluating elective single embryo transfer (eSET) in good prognosis patients are were illustrative of the potential advantage of blastocyst transfer; Milki et al. randomized 611 women less than 36 years of age with at least two good-quality embryos to eSET or double embryo transfer, of which $97.2 \%$ underwent transfer on day 2 or 3 (the majority on day 2). The implantation rate for the first eSET was $33.6 \%$ (37).

In contrast, Gardner et al. randomized 48 women with similar baseline characteristics and at least 10 follicles $>12 \mathrm{~mm}$ in diameter on the day of $\mathrm{hCG}$ administration to elective single or double day 5 blastocyst stage embryo transfers ( $\mathbf{3 8}$ ). A host of prospective randomized trials have compared cleavage to extended stage embryo transfer, the majority of which demonstrated improved outcomes with the latter, one of the few trials which reported lower live birth rates with blastocyst transfer noted similar implantation rates for both groups. Interestingly, all blastocyst transfers in this study were performed on day 6, which may be a confounding variable. Indeed, others have demonstrated that day 5 blastocysts may be better synchronized with endometrial development than more slowly developing embryos transferred on day 6 , resulting in higher pregnancy rates with day 5 transfer (39).

Arecent Cochrane meta-analysis found no evidence of a difference in live birth or pregnancy outcomes between Days 2 - 3 and 5-6 embryos transfers ,Moreover, blastocyst transfer was associated with an increase in failure to transfer any embryos in a cycle and a decrease in embryo freezing rates 
(40). A fundamental error performed in many randomized controlled trial (RCTs) comparing blastocyst transfer over cleavage-stage transfer was that the number of embryos replaced was unequal in the two groups, with more embryos transferred in the cleavage-stage group. Therefore, by allowing by definition (at randomization point) more embryos to be replaced in the Day 3 group, then the relative advantage of blastocysts having better correlation with genetic quality disappears (41).

If we consider that accumulating evidence suggests that in top-quality cleavage-stage embryos up to $60 \%$ might be aneuploidy, whereas in topquality blastocysts this percentage might reach $30 \%$ (42), it is obvious that the fewer embryos transfer ; single-embryo transfer ; the higher the chance to transfer genetically abnormal embryos when earlier developmental stage embryos are transferred. Thus the transfer of extra embryos in the Day 3 group compared with Day 5 group increases the probability of transferring aneuploid embryo and thereby reducing the selection bias in early embryonic developmental stages (cleavage stages).

Since the aforementioned meta-analysis emerged, two more studies have been published increasing by $40 \%$ the study population (43). Perhaps more telling are the results of prospective randomized trials comparing elective single cleavage to blastocyst stage embryo transfer.

Papanikolaou et al. randomly assigned 351 women under 36 years of age to transfer of a single cleavage stage (day 3 ) or blastocyst stage (day 5) embryo, The study was terminated after an interim analysis demonstrated significantly higher ongoing pregnancy rates $(58 \%$ vs. $41 \%, \mathrm{P}=0.02 ; 95 \% \mathrm{CI}$ $1.06-2.66)$ and live birth rates (56 \% vs. $38 \%, \mathrm{P}$ $=0.01 ; 95 \%$ CI 1.09-2.18) per embryo transfer procedure in the blastocyst group Subsequently (44). Zech and coworkers performed a similar study of 227 women 36 years of age undergoing a first or second IVF cycle, resulting in 5 fertilized oocytes (45).

Guerif and coworkers recently completed a prospective study of 478 couples assigned to day 2 eSET or single blastocyst transfer on day 5 or 6 . It is important to note that patients were assigned on a "voluntary basis" which represents a confounding variable. Nevertheless, the delivery rate per fresh embryo transfer was again significantly higher after single blastocyst transfer (36.7 \% vs. 25.1
$\%, \mathrm{P}<0.01)$. It is interesting to note that a recent meta-analysis of live birth rates after elective single cleavage stage embryo transfer in prospective randomized trials described a live birth rate of $26.7 \%(46)$.

Two recent meta-analyses addressing this issue with different designs and reaching different conclusions have been published. An updated Cochrane review evaluated randomized trials of early cleavage (day 2/3) versus blastocyst (day $5 / 6)$ stage transfers. Sixteen of the 45 identified trials met inclusion criteria and were analyzed. Interestingly, there was no difference in live birth rates per couple in seven randomized clinical trials (day 2/3: $34.3 \%$ vs. day 5/6: $35.4 \%$; OR 1.16 , $95 \%$ CI $0.74-1.44)(47)$. This phenomenon held true for "good prognosis" patients as well. There was also a greater likelihood of having no embryos to transfer in the blastocyst. Comparative implantation rates (IR) resulting in live birth after elective single cleavage (eSET) or blastocyst stage (eBT) embryo transfer (48).

Many published studies that debate the correlation of morphological features with pregnancy rates can be found in the literature (49). It is now understood that a disturbingly large proportion of morphologically normal Day 3 embryos are chromosomally abnormal, thus contributing to the $80 \%$ to $90 \%$ rate of implantation failure post transfer that is observed in cleavage stage protocols (50). While the transfer of Day 5 embryos cannot ensure the absence of chromosomal abnormality, it have been demonstrated that, at least in women older than $36 y$ years, the incidence can be reduced from $59 \%$ on Day 3 to $35 \%$ in Day 5 blastocysts (51).

\section{Conclusion:}

The introduction of sequential media that takes into account the changing metabolic requirement of the embryo, as it develops from the zygote to the blastocyst stage, allows extended culture. Blastocyst transfer should enable transfer of fewer but higher quality embryos resulting in increased implantation rates. This would maintain a high pregnancy rate while controlling the multiple pregnancy rates . Reasons for higher success rates with blastocyst are mainly related to embryo selection process. Embryos selected for transfer on Day 5 are healthier and carry a lower risk of being aneuploidy, thereby increasing patient's chance of achieving an ongoing pregnancy (52). Although blastocyst transfer has been shown to be beneficial 
in good prognosis patients, and similar benefits were not seen in an unselected group.

In conclusion, this study has shown that in younger patients with good ovarian response, extended culture to Day 5 can be offered, as blastocyst transfer is found to have good clinical pregnancy rates. The good clinical pregnancy and implantation rates observed will confidently allow transfer of not more than two good quality blastocyst and allow women to enjoy the benefits of limiting numbers for transfer.

\section{References:}

1. Racowsky C, Jackson KV, Cekleniak NA, Fox JH, Hornstein MD, Ginsburg ES. (2000): The number of eight- cell embryos is a key determinant for selecting day 3 or day 5 transfers. Fertil Steril; 73(3):558-64.

2. Alper MM, Brinsden P, Fischer R, Wikland M. (2001): To blastocyst or not to blastocyst? That is the question. Hum Reprod; 16(4):617-9.

3. de los Santos MJ, Mercader A, Galán A, Albert C, Romero JL, Pellicer A. (2003):Implantation rates after two, three, or five days of embryo culture. Placenta; 24 Suppl B: S13-9.

4. Graham J, Han T, and Porter R, et al. (2000): Day 3 morphology is a poor predictor of blastocyst quality in extended culture. Fertil Steril.; 74:495-7.

5. Papanikolaou EG, D'haeseleer E, Verheycn G, Van de Velde H, Camus M, Van Steirteghem A, Devroey P, Tournaye H. (2005): Live birth rate is significantly higher after blastocyst transfer than after cleavage-stage transfer when at least four embryos are available on day 3 of culture. A randomized prospective study. Human Reproduction 2005; 20(11):3198-203.

6. Waters A-M, Dean JH, Sullivan EA. (2006): Assisted reproduction technology in Australia and New Zealand 2003. AIHWCat.No. PER 31 Sydney Assisted Reproduction. Series 9. AIHW National Perinatal Statistics Unit, 2006.

7. Papanikolaou EG, Camus M, Kolibianakis EM, Van Landuyt L, Van Steirteghem A, Devroey P. (2006): In vitro-fertilization with single blastocyst-stage versus single cleavage-stage embryos. N Engl J Med; 354(11): 1139-46.

8. Fragouli E, Lenzi M, Ross R, et al.( 2008):Comprehensive molecular cytogenetic analysis of the human blastocyst stage. Hum Reprod. 23:2596-608.
9. Levens E, Whitcomb B, and Hennessy S, et al. (2008): Blastocyst development rate impacts outcome in cryopreserved blastocyst transfer cycles. Fertil Steril.; 9012: 2138-43.

10. Kolibianakis EM, Zikopoulos K, Verpoest W, Camus M, Joris H, and Van Steirteghem AC, et al. (2004): Should we advise patients undergoing IVF to start a cycle leading to a day 3 or a day 5 transfers? Hum Reprod; 19(11): 2550- 4.

11. Bungum M, Bungum L, Humaidan P and Yding Andersen C. (2003): Day 3 versus day 5 embryo transfer: a prospective randomized study. Reproductive Biomedicine Online April 2003; 7(1):98-104.

12. Coskun S, Hollanders J, Al-Hassan S, Al-Sufyan H, AlMayman H, Jaroudi K. (2000): Day 5 versus day 3 embryo transfer: a controlled randomized trial. Human Reproduction; 15(9):1947-52.

13. Emiliani S, Delbaere A, Vannin A, Biramane J, Verdoodt M, Englert Y, Devreker F. (2003): Similar delivery rates in a selected group of patients, for day 2 and day 5 embryos both cultured in sequential medium: a randomized study. Human Reproduction; 18(10):2145-50.

14. Staessen C, Platteau P, Assche E, van, Michiels A, Tournaye H, Camus M, et al. 2004Comparison of blastocyst transfer with or without preimplantation genetic diagnosis for aneuploidy screening in couples with advanced maternal age: a prospective randomized controlled trial. Hum Reprod. ;19:2849-58.

15. Devreker F, Delbaere A, Emiliani S, Van den Bergh M, Biramane J Englert Y. (2000): Prospective and randomized comparison between transfer on day 2 or day 5 for patients with more than four IVF attempts. ESHRE. 2000:P135.

16. Gardner DK, Surry E, Minjarez D, Leitz A, Stevens J, Schoolcraft WB. (2004): Single blastocyst transfer a prospective randomised trial. Fertility and Sterility 2004; 81:551-5.

17. Milki A, Hinckley M, Gebhardt J, Dasig D, Westphal L, Behr B. Accuracy of day 3 criteria for selecting the best embryos. Fertil Steril. 2002; 77:1191-5.

18. Braude P, Bolton V, and Moore S. (1998): Human gene expression first occurs between the four and eight-cell stages of preimplantation development. Nature 1988; 332:459-61.

19. Schoolcraft WB, Gardner DK. (2001): 
Blastocyst versus day 2 or 3 transfer. Seminars in Reproductive Medicine 2001; 19: 259-268.

20. Hreeinsson J, Rosenlund B, Fridstrom M, Ek I, Levkov L, Sjoblom P, Hovatta O. (2004): Embryo transfer is equally effective at cleavage stage and blastocyst stage: a randomized prospective study. European Journal of Obstetrics Gynecology and Reproductive Biology 2004; 117:194-200.

21. Valbuena D, Martin J, dePablo J, Remohi J, Pellicer A, Simon C. (2001): Increasing levels of estradiol are deleterious to embryonic implantation because they affect the embryo. Fertility and Sterility 2001; 76(5):962-8.

22. Blake D, Proctor M, Johnson N, et al. (2005): Cleavage stage versus blastocyst stage embryo transfer in assisted conception. Cochrane Database Syst Rev. ;( 4), Art No: CD002118. Pub 2.

23. Technology and the Practice Committee of the American Society for Reproductive Medicine. (2008): Guidelines on number of embryos transferred. Fertil Steril.; 90 Suppl 3:S163-4.

24. Fanchin R, Ayoubi JM, Righini C, et al. (2001): Uterine contractility decreases at the time of blastocyst transfers. Human Reproduction; 16:1115-9.

25. Karaki RZ, Samarraie SS, Younis NA, Lahloub TM, Ibrahim MH. (2002): Blastocyst culture and transfer: a step toward improved in vitro fertilization outcome. Fertility and Sterility 2002; 77:114-8.

26. Gardner DK, Lane M. (2003): Blastocyst culture. Clinical Obstetrics and Gynecology b; 46:231-8.

27. Schoolcraft W, Fragouli E, Stevens J, et al. (2010): Clinical applications of comprehensive chromosomal screening at the blastocyst stage. Fertil Steril.; 94:1700-6.

28. Bukulmez Bukulmez O, REhman KS, Langely M, Carr BR, Nackley AC, Doody KM, Doody KJ. (2007): Precycle administration of GnRH antagonist and micro dose HCG deceases clinical pregnancy rates without affecting embryo quality and blastulaion. Reproductive Medicine Online 2006; 13: 465-75.

29. Hamberger L, Hardarson T, Nygren KG. (2005): Avoidance of multiple pregnancies by use of single embryo transfer. Minerva Ginecologica 2005; 57:15-9.
30. Jain JK, Boostanfar R, Slater CC, Francis MM, Paulons RJ. (2004): Monozygotic twins and triplets in association with blastocyst transfer. Journal of Assisted Reproductive Genetics 2004; 21(4):103-7.

31. Botros L, Sakkas D, Seli E. (2008): Metabolomics and its application for non-invasive embryonic assessment in IVF. Mol Hum Reprod. 14:67990 .

32. Neuber E, Mahutte N, Arici A, Sakkas D. Sequential embryo assessment outperforms investigators-driven morphological assessment at selecting a good quality blastocyst. Fertil Steril. 2006;85:794-6.

33. Levron J, Shulman A, Bider D, Seidman D, Levin T, Dor J. (2002): A prospective randomized study comparing day 3 with blastocyst-stage embryo transfer. Fertility and Sterility 2002; 77:1300-1.

34. Levitas E, Lunenfeld E, Hackmon-Ram R, Sonin Y, HarVardi I, Potashnik G. (2004): A prospective, randomized study comparing blastocyst versus 48-72 h embryo transfer in women failed to conceive three or more in-vitro fertilization treatment cycles. Abstract from the 57th Annual Meeting of ASRM. 2001.

35. Van der Auwera I, Debrock S, Spiessens C, Afschrift H, Bakelants E, and Meuleman C, et al. (2002): A prospective randomized study: day 2 versus day 5 embryo transfer. Hum Reprod; 17(6):1507-12.

36. Mangalraj AM, Muthukumar K, Aleyamma T, Kamath MS, George K. (2009): Blastocyst stage transfer vs cleavage stage embryo transfer. J Hum Reprod Sci ; 2(1):23-6.

37. Milki A, Hinckley M, Westphal L, Behr B. (2004): Elective single blastoyst transfer. Fertility and Sterility; 81:1697-1698.

38. Gardner DK, Surrey E, Minjarez D, Leitz A, Stevens J, Schoolcraft WB. (2004): Single blastocyst transfer: a prospective randomized trial. Fertil Steril; 81(3):551-5.

39. Glujovsky D, Farquhar C. (2016): Cleavagestage or blastocyst transfer: what are the benefits and harms? Fertil Steril.; 106:244-50.

40. Sigalos G, Triantafyllidou O, Vlahos NF. (2016): Novel embryo selection techniques to increase embryo implantation in IVF attempts. Arch Gynecol Obstet.; 294:1117-24. 
41. Kaser DJ, Racowsky C. (2014): Clinical outcomes following selection of human preimplantation embryos with time lapse monitoring: a systematic review. Hum Reprod Update.; 20:617-31.

42. Gardner DK, Meseguer M, Rubio C, Treff NR. (2015): Diagnosis of human preimplantation embryo viability. Hum Reprod Update; 21:72747.

43. Moragianni VA, Penzias AS (2010): Cumulative live-birth rates after assisted reproductive technology. Curr Opin Obstet Gynecol.; 22:18992.

44. Papanikolaou E, Fatemi H, Venetis C, et al. (2010): Monozygotic twinning is not increased after single blastocyst transfer compared with single cleavagestage embryo transfer. Fertil Steril.; 93:592-7. 59.

45. Zech NH, Lejeune B, Puissant F, Zech H, Vanderzwalmen S, Vanderzwalmen P. (2005): Single embryo transfer: selection on day 3 or day 5? A prospective observational study. Fertil Steril. ; 84(Suppl 1):S266.

46. Malizia BA, Hacker MR, Penzias AS. (2009): Cumulative live-birth rates after in vitro fertilization. $N$ Engl J Med.; 360:236-43,
47. Maheshwari A, McLernon D, Bhattacharya S (2015): Cumulative live birth rate: time for a consensus? Hum Reprod. ; 30:2703-7.

48. Tiras B, Cenksoy PO. (2014): Practice of embryo transfer: recommendations during and after. Semin Reprod Med.; 32:291-6.

49. Mantikou E, Youssef MA, Wely M, van, AlInany HG, Repping S, Mastenbroek S.( 2013): Embryo culture media and IVF/ICSI success rates: a systematic review. Hum Reprod Update. 19:210-20.

50. Guzoglu N, Kanmaz HG, Dilli D, Uras N, Erdeve O, Dilmen U. (2012): The impact of the new Turkish regulation, imposing single embryo transfer after assisted reproduction technology, on neonatal intensive care unit utilization: a single center experience. Hum Reprod. 27:2384-8.

51. Schwarzler P, Zech H, Auer M, Pfau K, Gobel G, Vanderzwalmen P, Zech N. (2001): Pregnancy outcome after blastocyst transfer as compared to early cleavage stage embryo transfer. Hum Reprod. 19:2097-102.

52. Blake DA, Proctor M, Johnson NP. (2004): The merits of blastocysts versus cleavage stage embryo transfer: a Cochrane review. Hum Reprod. 19:795-807. 